\title{
Channel Optical Waveguides and Directional Couplers in GaAs-Imbedded and Ridged
}

\author{
S. Somekh, E. Garmire, A. Yariv, H. L. Garvin, and R. G. Hunsperger
}

\begin{abstract}
Two-channel imbedded directional couplers were fabricated with proton implantation, yielding complete light transfer in $2 \mathrm{~mm}$. Ridged channel guides were fabricated by ion-micromachining epitaxial layers, and a method of directional coupling was demonstrated.
\end{abstract}

The development of useful integrated optics circuits requires channel waveguides of small dimensions fabricated with close tolerances. We have investigated ways of making channel guides in GaAs, both imbedded guides and ridged. In addition to single channel guides, we have investigated the coupling properties of dual channel imbedded guides and have made a bridged structure for coupling ridged guides. Finally, we look at the use of a directional coupler as a light switch.

The imbeddèd channels were fabricated in GaAs by $300-\mathrm{keV}$ proton implantation ${ }^{\mathbf{1}}$ in a fashion similar to the fabrication of a multichannel directional coupler. $^{2}$ Bombardment causes defect centers in the material, which trap the free carriers. This compensation of the free carriers eliminates their negative plasma contribution to the index of refraction and thus increases the index. For the samples used here (N-type GaAs $\sim 2 \times 10^{18} / \mathrm{cc}$ ) the index increase is $\Delta n \sim 0.005$. The channel guides were formed by implanting the protons through a proton-resistant gold mask, $2 \mu \mathrm{m}$ thick. The pattern was ion-machined into the gold through a photoresist mask. The implanted guides have cross-sectional dimensions of 3 $\mu \mathrm{m} \times 3 \mu \mathrm{m}$ and are single mode in both confinement directions, an important requirement for many optical circuit elements. We measured a single channel loss of $6 \mathrm{~cm}^{-1}$ for the first order mode; this loss is determined by the particular implanting and annealing conditions and is comparable with losses in planar implanted guides. ${ }^{1}$ The mode profiles and losses were studied in a fashion similar to Ref. 1 . We have found that ion implantation provides an excellent means of making smooth imbedded optical circuits.

The first three authors named are with the California Institute of Technology, Pasadena, California 91109; the other authors are with Hughes Research Laboratories, Malibu, California 90265 except S. Somekh who is now with Bell Telephone Laboratories, Murray Hill, New Jersey 07974.

Received 20 July 1973.

CLEA paper 12.4 .
The statistical nature of implantation and the annealing help to smooth out any mask imperfections so that the presence of channel walls does not substantially increase the losses. Furthermore, the protons directly copy complex circuits from a gold mask into the GaAs crystal.

When two parallel channel guides are sufficiently close, energy can be coupled from one to another. This forms a dual channel directional coupler. The overlap of the mode profiles in the two guides determines the strength of the coupling. If all the light is originally fed into one channel and the two modes have the same propagation constants, the power oscillates between channels in a sinusoidal fashion. The power flow in the two guides as a function of propagation distance $z$ is ${ }^{3}$

$$
\begin{aligned}
& P_{0}^{(z)}=\cos ^{2} K z e^{-\alpha z}, \\
& P_{1}^{(2)}=\sin ^{2} K z e^{-\alpha z},
\end{aligned}
$$

where $K$ is the coupling between guides and $\alpha$ is the exponential loss constant in each guide.

Figure 1 illustrates three pairs of such directional couplers, made of implanted guides imbedded at the surface with cross sections of about $3 \mu \mathrm{m} \times 3 \mu \mathrm{m}$, an index discontinuity of about $\Delta n \simeq 0.005$, and a separation of $3 \mu \mathrm{m}$ between the guides. On the left is the input plane of the device, and the black dot indicates the point at which the input light was focused. On the right is a series of scans of the corresponding light distribution at the output plane, taken by the setup described in Ref. 1 . Let us confine our attention to the directional coupler at the center. The second row from the top shows that when the input light is coupled into the channel on the right, it emerges at the output plane from both channels. Similarly, the second row from the bottom indicates that the light also emerges from both channels when it is coupled into the left channel. Since the intensity of the emerging light from both channels is about equal, we can write for this sample, $K L \simeq \pi / 4$; since $L=1 \mathrm{~mm}, K \simeq 0.79 \mathrm{~mm}^{-1}$. By increasing the 


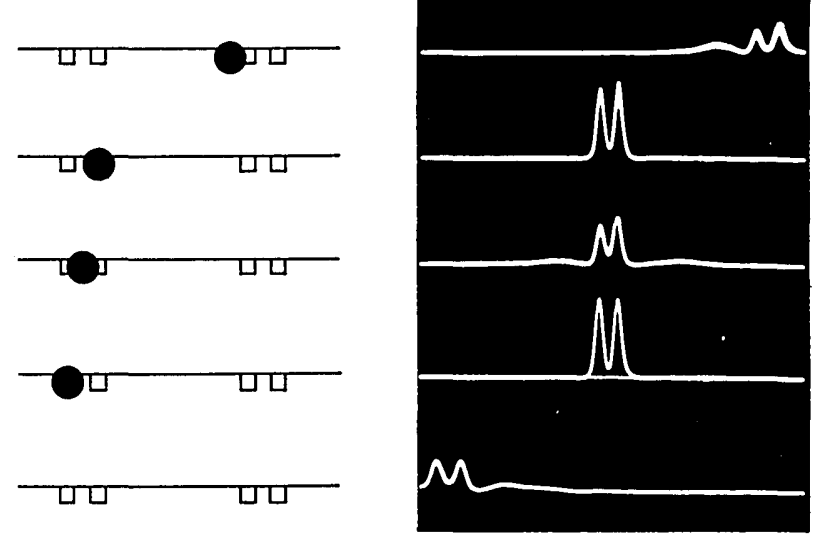

Fig. 1 Left: Each horizontal row corresponds to the input plane of the crystal with three directional coupler pairs. The black dot is the position of the input laser beam. Right: The corresponding intensity plots in the output face of the crystal.

The length of the crystal is $1 \mathrm{~mm}$.
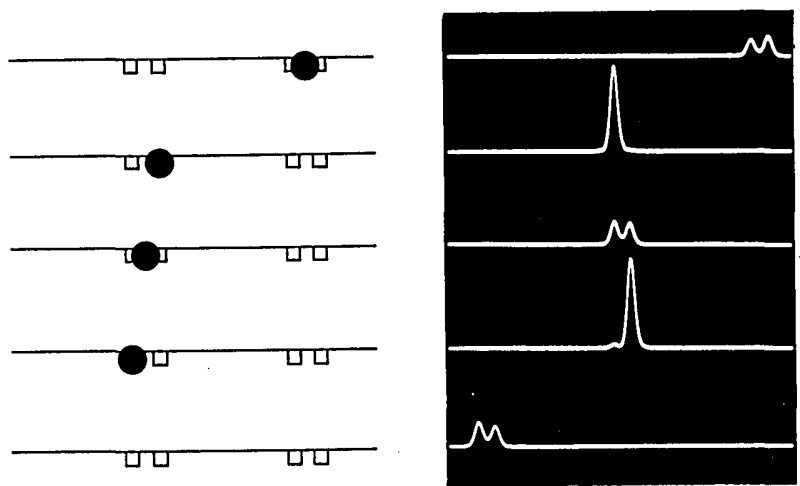

Fig. 2. Left: Each horizontal row corresponds to the input plane of the crystal with three directional coupler pairs. The black dot is the position of the input laser beam. Right: The corresponding intensity plots in the output face of the crystal. The length of the crystal is $2.1 \mathrm{~mm}$.

length of the sample by about a factor of 2 , a complete transfer of power should be observed since $K L$ $=\pi / 2$. This is shown in Fig. 2. The second row from the top describes a situation in which light coupled into the left channel emerges from the one on the left, and the second row from the bottom shows the transfer of power from left to right. The observed $2-\mathrm{mm}$ coupling length is consistent with previous measurements of coupling strength of implanted guides, taking into account the change in channel dimensions, and is in agreement with a calculation based on the dielectric discontinuity and channel dimensions. The 2-mm length is convenient for many optical circuit applications.

Let us now consider ridged channels. These can be fabricated by removing the superfluous sections of a planar epilayer. In our case this was done by starting with epitaxial films of GaAs (high resistivity grown on low resistivity substrates) and then ionmicromachining through a photoresist mask. The surface of the photoresist is replicated in the epitaxial layer by the milling action of the ion beam. ${ }^{4} \mathrm{~A}$ diagram and photograph of such ridged guides can be seen in Fig. 3(a).

Because of the large dielectric discontinuity, the scattering losses in this guide are very sensitive to the smoothness of its walls as well as to that of the top surface. For example, a $3-\mu \mathrm{m}$ wide waveguide in GaAs will suffer roughly a loss of $4 \mathrm{~cm}^{-1}$ when the roughness of the walls is $500 \AA \mathrm{rms}$. This rough estimate ${ }^{5}$ based on the Rayleight criterion indicates that special care has to be taken in the fabrication of these guides. Smooth channels were fabricated by exposing the photoresist with a holographically prepared mask ${ }^{4}$ and ion-polishing the sample after the ion-matching. Figure 4 shows such a channel (1.4
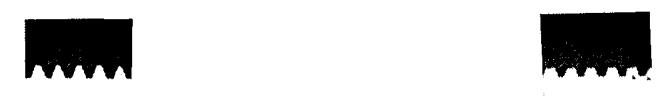

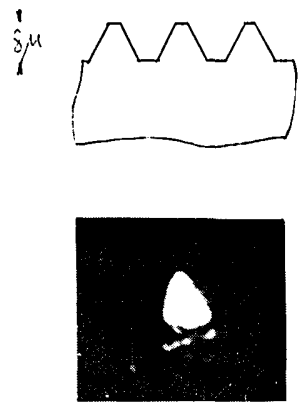

(a)
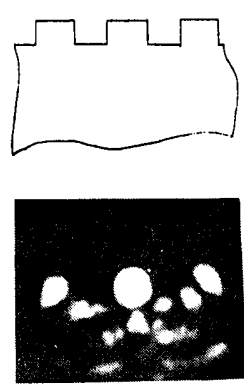

(b)
Fig. 3. Directional coupler for ridged guides fabricated by ionmatching in a GaAs epilayer. (a) Isolated channels; (b) coupled channels.

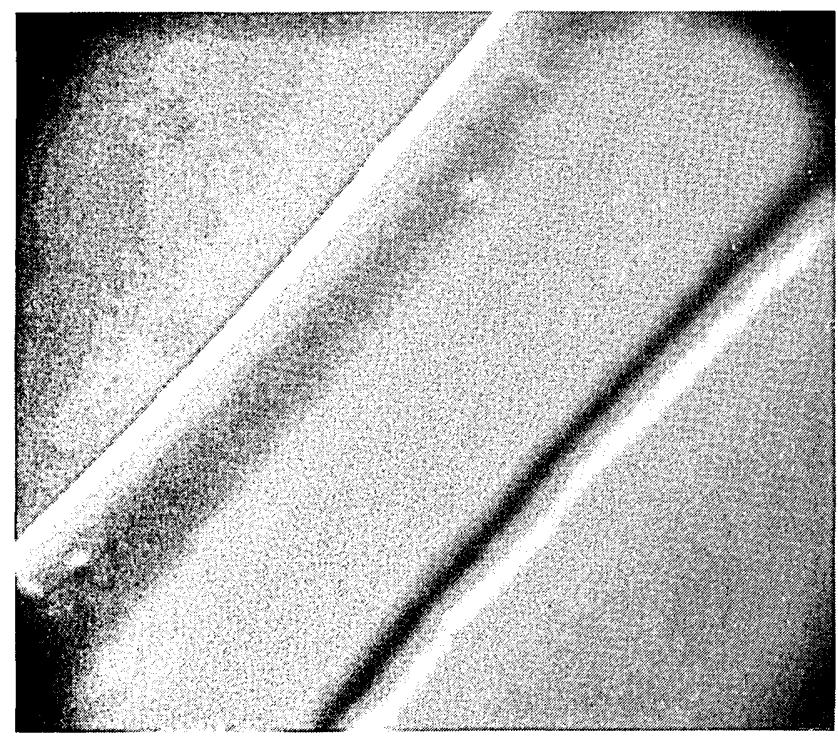

Fig. 4. SEM of channel waveguide fabricated by using holographically preparêd mask and ion-polishing. (The guide is $1.4 \mu \mathrm{m}$ high and $2 \mu \mathrm{m}$ wide.) 
$\mu \mathrm{m}$ high, $2 \mu \mathrm{m}$ wide). The edge deviations appear to be less than a few hundred angstroms. This also suggests that instead of cleaving, a smooth edge can be ion-machined to make good terminations to imbedded or ridged channel guides. Our ion-machining seems to be sufficiently smooth to fabricate mirrors for semiconductor lasers and may even allow curved mirrors in order to increase the cavity $Q$.

Let us consider directional couplers in GaAs made with ridged channels. In this case, the modes are well confined in the plane of the guide because of the large dielectric discontinuity at the ridge-air interface. Thus two closely spaced ridged guides will have negligible coupling, since they have no overlapping modes. To increase the coupling between channels, only a partial removal of the epilayer between them was performed. A comparison of total and partial removal of epilayer material between channels is shown in Fig. 3. In Fig. 3(a) we see the cross section of a high resistivity GaAs epilayer (about $8 \mu \mathrm{m}$ thick) that was machined down to the substrate, forming a large number of isolated channels. Light entering into one channel emerged at the other end of the sample from that channel only (bottom photograph). However, when the same epilayer was only partially machined [Fig. 3(b)], light entering into one guide emerged from the adjacent guides as well. The strength of this coupling depends on the thickness of the epilayer bridge between channels. The coupling between two ridged channels can also be increased by filling the region between them with another material with a slightly lower refractive index, for example, by sputtering.

One of the most exciting applications of the directional coupler is the switch modulator in which the amount of light coupled from one channel to the other is controlled electrooptically. Such a device can be used to modulate the light propagating in one guide or to switch light from one channel to another. The latter property is an important means for multiplexing (or demultiplexing) two signals into one or for switching light in an optoelectronic switchboard of an optical communication network.

We shall compare the directional coupler modulator with the polarization modulator. ${ }^{6}$ The latter requires the total phase difference between TE and TM modes to change from 0 to $\pi$ with the application of a field to achieve $100 \%$ modulation. The required difference in refractive index between TE and TM modes with field applied is then $\delta n=\pi / k_{0} L$, where $L$ is the length of the modulator and $k_{0}$ is the free space propagation vector.

Consider an example of directional coupler modulator: The channel cross section is $3 \mu \mathrm{m} \times 3 \mu \mathrm{m}$ and the separation is $3 \mu \mathrm{m}$ with $\Delta n=0.01$. At $1.15 \mu \mathrm{m}$ we have $K=1.55 \mathrm{~cm}^{-1}$. Full transfer of power from one channel into the other occurs in a modulator length $L=(\pi / 2)(1 / \mathrm{K}) \sim 1 \mathrm{~cm}$. If the coupling coefficient is increased (by changing the dielectric discontinuity with the electrooptic effect) to a new value $K_{1}=2 K$, the light will be coupled back into the original channel, resulting in $100 \%$ modulation. ${ }^{7}$
The coupling coefficient can be varied by applying field to the section between the two guides or alternatively by applying the field to the guides. To obtain the above value of $K_{1}$ the dielectric discontinuity of the guide has to be reduced from $\Delta n=0.01$ to $\Delta n=0.007$. Thus the change in the index of refraction required for switching is

$$
\delta n \simeq 0.003=52\left[\pi /\left(k_{0} L\right)\right]
$$

or $52 \times$ the dielectric change (or electric field) needed for an electrooptic polarization modulator of the same length. This requires a substantial increase in the power needed to drive the modulator, compared to polarization modulation, an undesirable requirement for circuit applications.

A different scheme for a directional coupler modulator involves applying a field to only one channel. The phase velocity synchronism of the fields in the two channels is spoiled thereby, and the effectiveness of coupling can be reduced so that all the light comes out the original channel instead of the opposite channel. When the light propagating in the two channels differs in propagation constant by $\Delta \beta$, the power flow obeys the following equation ${ }^{3}$ :

where

$$
\begin{gathered}
P_{0}(z)=\cos ^{2} S z+\left[(\Delta \beta / 2)^{2} / S^{2}\right] \sin ^{2} S z \\
P_{1}(z)=\left(K^{2} / S^{2}\right) \sin ^{2} S z
\end{gathered}
$$

$$
S^{2}=K^{2}+(\Delta \beta / 2)^{2} \text {. }
$$

The value $\Delta \beta$ needed to destroy the original coupling $(K L=\pi / 2)$ is given by $S L=\pi$ or $\Delta \beta L=\sqrt{ } 3 \pi$, which implies $\delta n \sim \sqrt{ } 3 \pi / k_{0} L$, which is larger by only a factor of $\sqrt{ } 3$ than the value required for polarization modulation. Thus the directional coupler switch in which a field is applied to only one channel compares favorably . with a polarization modulator and should be developed for use in optical circuits.

A directional coupler can be made polarization sensitive by choosing an appropriate guiding plane, for example (100) in GaAs, so that the application of field causes a certain $\delta n$ for TE modes but $\delta n=0$ for TM modes (or vice versa). Thus, in our example, before the application of the field TE as well as TM modes are coupled from one guide to the other. However, with the application of the field, the coupling of the TE mode is destroyed, while the coupling of the TM mode remains the same. Such a device with a dc electric field is capable of separating the two polarizations of an incoming optical signal into two different channels. As such it can turn a polarization modulator (or a mode converter) into a directional coupler switch. This composite device will have a low modulating power together with a directional coupler switch capability. The polarization-sensitive directional coupler can also be used, by operating it in reverse to the manner described above, to combine two separate channels with different polarizations into one output channel (multiplex). 
In conclusion, we have studied some methods for making channel guides and directional couplers in GaAs. Other methods for making imbedded guides with diffusion, or by covering ridged guides, remain to be tried. Chemical etching or direct growth through masks are other possibilities for making ridged guides. Finally, we have compared directional coupler modulation with polarization modulation and found that application of a field to only one channel is the most favorable configuration for a directional coupler switch.

The research reported in this paper was partially supported by the Army Research Office, Durham, North Carolina.

\section{References}

1. E. Garmire, H. Stoll, A. Yariv, and R. G. Hunsperger, Appl. Phys. Lett. 21, 87 (1972).

2. S. Somekh, E. Garmire, A. Yariv, H. L. Garvin, and R. G. Hunsperger, Apl. Phys. Lett. 22, 46 (1973).

3. See for example, W. H. Louisell, Coupled Modes and Parametric Electronics (Wiley, New York, 1960).

4. H. L. Garvin, E. Garmire, S. Somekh, H. Stoll, and A. Yariv, Appl. Opt. 12, 455 (1973).

5. P. K. Tien, Appl. Opt. 10, 2395 (1971).

6. D. Hall, A. Yariv, and E. Garmire, Opt. Commun. 1, 403 (1970).

7. E. A. J. Marcatili, Bell Syst. Tech. J. 48, 2071 (1969).

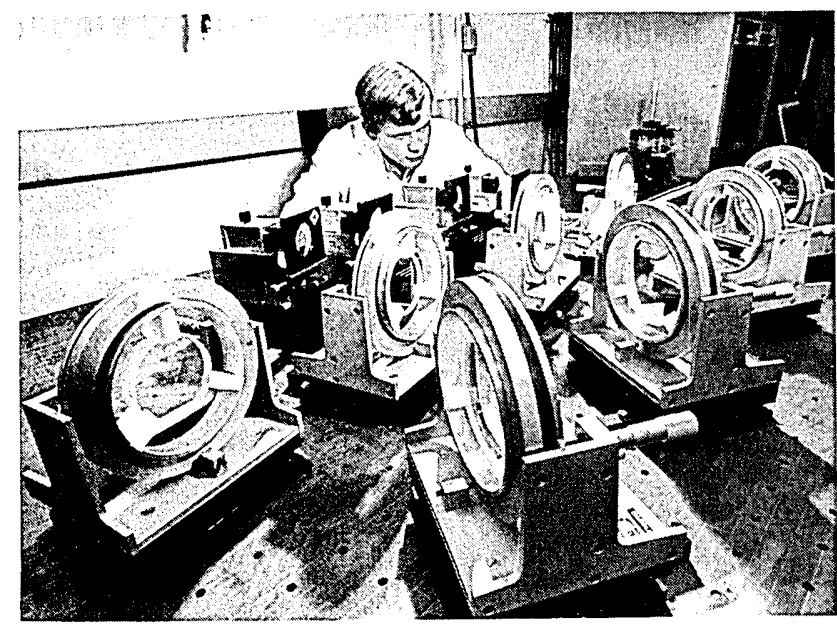

Sandia Laboratories scientists have begun the Atomic Energy Commission's first multiple-beam laser experiments to obtain fundamental data relevant to production of energy by laser-induced controlled thermonuclear fusion. The four-beam neodymium-glass laser is presently producing about $200 \mathrm{~J}$ in bursts lasting 2 nsec, subjecting $200 \mu \mathrm{m}$ diam deuterated polyethylene pellets to a peak power of about 100-billion W. Output of the system will be scaled up gradually until a total energy of approximately $500 \mathrm{~J}$ in a $2 \mathrm{nsec}$ pulse is reached. Subnanosecond experiments will then begin, with pellets being irradiated with pulses lasting 200-500 psec. Power of these pulses would approximate 1-trillion W. The laser system consists basically of a small neodymium glass laser whose pulse is passed through four larger neodymium glass amplifiers, split into four beams, then passed through four more parallel amplifiers, with the beams finally terminating on the target pellet in an evacuated target chamber. Sandia, will use the new laser to study the potential of laser fusion. In the fusion studies, the laser will be used principally to fill the data gap between $50 \mathrm{~J}$ and $1000 \mathrm{~J}$. This is believed to be the first high energy system in the U.S. capable of generating more than two beams, although Russian experimenters have been operating a nine-beam laser in fusion experiments for several years. The Russian laser has a reported output of about $600 \mathrm{~J}$ in a $2 \mathrm{nsec}$ pulse. The four-beam laser system was designed and developed by Sandia's Laser Development Division headed by Eric D. Jones. Experiments are being conducted by the Laser Plasma Physics Division supervised by James E. Powell. 